\title{
8 ADHS und Teenager-Mutterschaft
}

Stephanie Bohne-Suraj und Olaf Reis

\subsection{Einleitung}

Während der letzten Jahrhunderte entsprach frühe Mutterschaft mal mehr und mal weniger der sozialen Norm, gleich, ob Deutschland oder andere Industriestaaten der Nordhalbkugel betrachtet werden. Mit dem zunehmenden Erstgebäralter jedoch rückt frühe Mutterschaft immer weiter von der weiblichen Durchschnittsbiographie ab. Die weibliche Normbiographie enthält mittlerweile ehemals „männliche“ Bestandteile, wie volle Schul- und Berufsausbildung, womit sich die Zeitpunkte verschiedener Entwicklungsübergänge verschoben haben. Deutschland gehört zu den Industrienationen, in denen diese Emanzipation der Frau relativ weit fortgeschritten ist. Zu den Folgen gehört, dass das durchschnittliche Alter bei Erstentbindung im Bundesgebiet bei 29, 8 Jahren bei verheirateten Frauen und bei 28 Jahren bei nicht verheirateten Frauen liegt. Damit entbinden Teenager-Mütter über ein Jahrzehnt früher als die meisten anderen, womit sich verstärkt die Frage nach den Folgen dieser extremer werdenden Außerzeitlichkeit stellt.

In den letzten Jahren wurde diesem Thema sowohl wissenschaftlich als medial steigende Aufmerksamkeit gewidmet. Die wissenschaftlichen Studien zu diesem Thema beschränken sich jedoch meist auf die Beschreibung der Situation junger Mütter. So wurde 2005 von der BZgA eine Studie zu TeenagerElternschaft veröffentlicht. Bei der Publikation mit dem Titel „Wenn Teenager Eltern werden ... Lebenssituation jugendlicher Schwangerer und Mütter sowie jugendlicher Paare mit Kindern" handelt es sich um eine rein qualitative Studie von Friedrich und Remberg auf Bundesebene, die über Interviewerhebungen die Vorstellungen und Erfahrungen schwangerer Jugendlicher zu den Bereichen Lebensplanung, Sexualität, professionelle Unterstützung und 
medizinische Versorgung beschreibt. Die Stichprobe dieser Studie blieb mit 43 schwangeren jungen Frauen, von denen 36 nach der Geburt ihrer Kinder erneut befragt wurden, relativ klein. Dennoch vermittelt das Lesematerial einen lebendigen Eindruck zu Umständen des Mutterseins als Jugendliche bzw. junge Erwachsene. Die Studie stellt u. a. fest, dass es den Interviewten trotz individuell hoher Motivation in der Regel nicht gelingt, ohne professionelle Hilfe allen Entwicklungsaufgaben gerecht zu werden.

Diesen Eindruck vermitteln auch Ergebnisse aus einzelnen Bundesländern. Die Studie „Teenager-Schwangerschaften in Sachsen. Angebote und Hilfebedarf aus professioneller Sicht" (Häußler-Sczepan et al. 2005) rückt dabei die Sichtweise von Experten in den Blickpunkt. Nach deren Meinung werden die breit gefächerten staatlichen, kommunalen und konfessionellen Hilfeangebote (ambulante Hilfen zur Erziehung, stationäre Jugendhilfe, Schwangerschaftskonflikt- und Schwangerenberatungsstellen) von den jungen Müttern zu wenig genutzt. Die Befragten vermuten, dass die durchaus vorhandenen Angebote von benachteiligten jungen Mädchen aus Scham und Angst nicht aufgesucht werden. In der Studie wird die Verknüpfung von medizinischer mit Sozial- und regelmäßiger Mütter-Beratung vorgeschlagen, wobei es vor allem darauf ankäme, berufliche Perspektiven für die Betroffenen zu schaffen. $\mathrm{Zu}$ ähnlichen Ergebnissen kommt eine Untersuchung zu Teenagerschwangerschaften in Berlin und Brandenburg (Wienholz 2007). Verschiedene Kommunen versuchen mit Projekten wie „Babybedenkzeit“, die nach Vorbildern aus den USA entwickelt wurden, Mädchen von früher Mutterschaft abzuhalten. In diesen Programmen werden Schülerinnen Computer-Babysimulationspuppen mit dem Auftrag übergeben, die Puppen zu „bemuttern“. Diese Interventionen gelten als sehr erfolgreich, da die Schülerinnen in praxi lernen, welchen Aufwand die 24-Stunden-Fürsorge um ein Baby bedeutet (Schöning 2004). Auch in Rostock wird ein solches Projekt praktiziert (http://www. babybedenkzeit.de/rostock.htm). Nach dem Wissen der Autoren gibt es bisher noch keine deutsche Studie, die die Wirkungen der Babybedenkzeit wissenschaftlich untersucht hätte.

Medial wurden und werden Aufsehen erregende Geschichten über „zu junge“ Mütter herausgebracht. Ein solcher Aufmacher war die Geschichte über eine mit 12 Jahren jüngste Mutter Deutschlands im März 2006.

\section{Im Hamburger Abendblatt wurde geschrieben}

„Es ist mitten in der Nacht, als Luiza (12, Name geändert) plötzlich ganz furchtbare Bauchschmerzen bekommt. Besorgt rufen ihre Eltern den Notarzt. Um genau 2.17 Uhr geht der Anruf bei den Rettern der Feuerwehr ein. Der Notarzt vermutet einen Blinddarmdurchbruch - und lässt das junge Mädchen aus der Wohnung der Eltern am Bargackerdamm (Bramfeld) ins Kinderkrankenhaus Wilhelmsstift einliefern. Dort wird die Zwölfjährige mit Ultraschall untersucht. Der Schock: Die Schmerzen sind Wehen, das Mädchen ist im neunten Monat schwanger!" 
Die Geschichte vermittelt deutlich das Bild von Frühreife und Vernachlässigung, wenn z. B. geschrieben wird: „Oft soll das Mädchen Partys gefeiert haben, wenn die Mutter und ihr Freund nicht zu Hause waren."

Auch anderen Medienberichten zufolge ist die Anzahl minderjähriger Mütter „dramatisch“ angestiegen. Fast durchgehend wird dabei die Teenager-Schwangerschaft problematisiert und als Ausdruck einer defizitären Entwicklung gesehen.

Der „dramatische Anstieg“ lässt sich nach den offiziellen Statistiken jedoch nicht feststellen. Im Vergleich mit anderen OECD-Staaten lag Deutschland 2002 mit einer Geburtenrate von 13,1 Geburten pro Tausend 15- bis 19-Jähriger im Mittelfeld. An der Spitze der Statistik stehen die USA mit einer Prävalenz von 52,1 Promille. Der hohe Anteil von minderjährigen Müttern in den USA ist vor allem deshalb über die letzten Jahrzehnte gestiegen, weil dort die jungen Frauen seit den 196oer Jahren die vorehelich geborenen Kinder nicht mehr an die Großfamilie weiter- bzw. zur Adoption freigeben. Stattdessen bleiben die Kinder zunehmend bei den alleinstehenden Müttern - die dann oftmals weder von der Familie noch von den Vätern unterstützt werden (Whitman et al. 2001).

Die relative Stabilität der Prävalenzen für frühe Mutterschaft in Deutschland ist jedoch auch durch die vergleichsweise liberalen Regelungen zum Schwangerschaftsabbruch bedingt. Darauf deuten die vom statistischen Bundesamt veröffentlichten Zahlen hin, nach denen es in Deutschland nur geringe Zunahmen früher Mutterschaft, wohl aber einen Anstieg minderjähriger Schwangerschaften und Schwangerschaftsabbrüche gibt ${ }^{1}$. Wie in den USA ist auch in Deutschland das Phänomen „frühe Mutterschaft“ regional sehr unterschiedlich verteilt. Während beispielsweise zwischen 2000 und 2002 die Gesamtgeburtenrate um 6,2\% sank, stieg zeitgleich die Anzahl adoleszenter Mütter durchschnittlich um $13 \%$ von anteilig o,6\% auf o, $8 \%$. Dabei stand eine $\mathrm{Zu}$ wachsrate von $10 \%$ in den alten Bundesländern einer fast doppelt so hohen Zunahme in den neuen Bundesländern $(18,7 \%)$ gegenüber. Großstädte scheinen Zentren dieser Entwicklung zu sein, denn in Berlin war ein Anstieg um $24 \% \mathrm{zu}$ verzeichnen. Laut UNICEF-Kinderstudie von 2006 führt Bremen mit 16,5 auf 1000 15-19-Jährige schwangere Mädchen diese Statistik an. In Mecklenburg-Vorpommern lag die Rate der Teenagerschwangerschaften bei 14/100o, in Baden-Württemberg hingegen nur bei 7,5 Promille (Bertram 2006).

Die Zu- und Abnahmen der Prävalenzen zeigen für Mecklenburg-Vorpommern keinen einheitlichen Trend (s. Abb. 7-9). Während zwischen 2000 bis 2003 ein Zuwachs um 8,7\% an Geburten Minderjähriger (bei einem Rücklauf aller Geburten um 4 \%) zu verzeichnen war, zeigt der Vergleich zwischen 2001 und 2004 einen Abfall um 9,9\% (bei einer Steigerungsrate von o,6\% für alle Geburten).

1 Von 2000-2003 erhöhte sich diese Rate bei Minderjährigen um 20,6 \% (Laue 2004). 


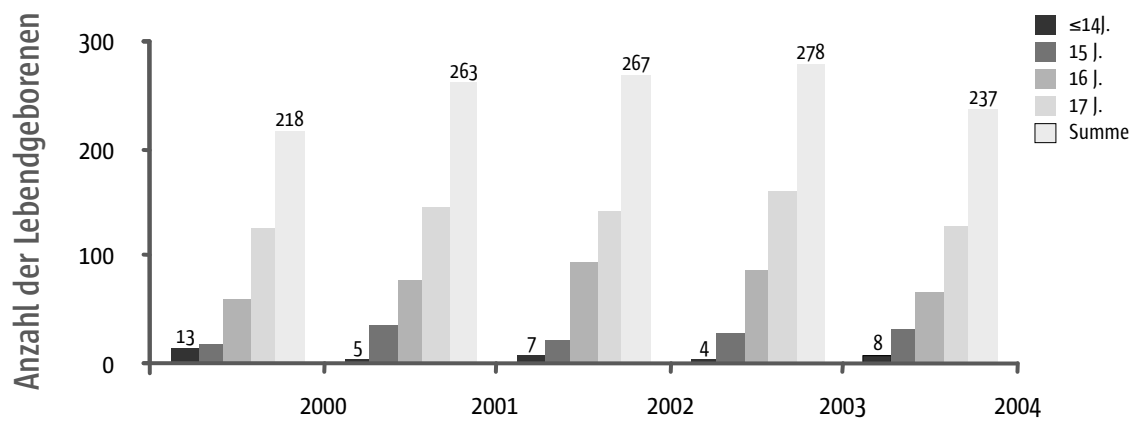

Abb. 7 Statistik Mecklenburg-Vorpommern 2000-2004, Lebendgeborene von minderj. Müttern (MiMü), absolute Zahlen

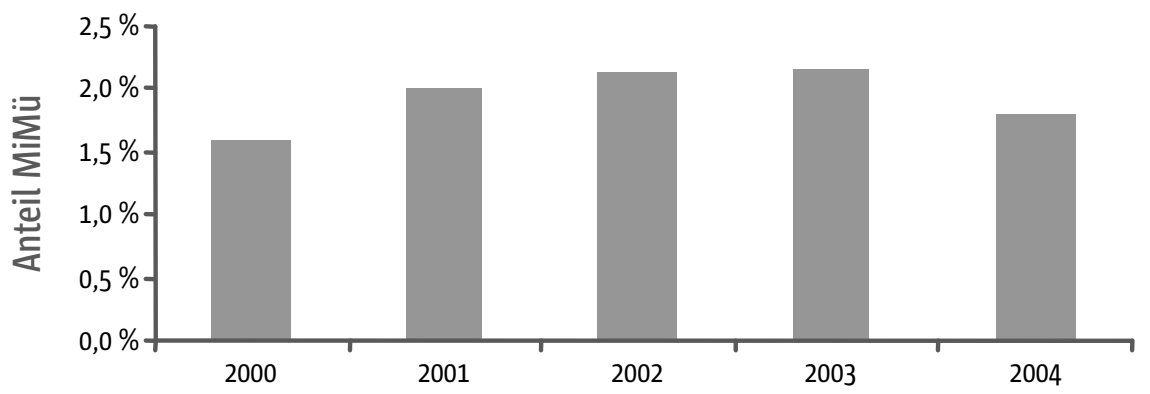

Abb. 8 Statistik Mecklenburg-Vorpommern 2000-2004, Anteil minderjähriger Mütter (MiMü) von Gesamtgeburten

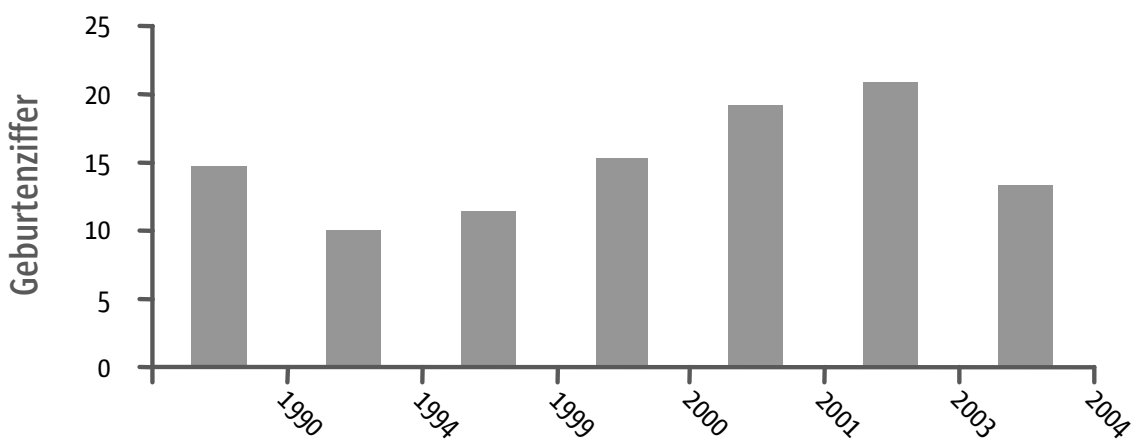

Abb. 9 Statistik Mecklenburg-Vorpommern, Geburtenziffern Lebendgeborene je 1000 Mädchen ausgewählter Jahrgänge

Im Vergleich zum gesamten Bundesgebiet (2000-2004) war die Anzahl der Schwangerschaftsabbrüche in Mecklenburg-Vorpommern mit -7,4\% sogar überdurchschnittlich rückläufig. Der Anteil minderjähriger Mütter an allen Geburten liegt in Deutschland bei 1,3\% (Statistisches Bundesamt 2005). Es handelt sich also um ein eher seltenes Phänomen. 


\subsection{Teenager-Mutterschaft als Entwicklungsrisiko}

Dennoch verdient die Teenagermutterschaft unsere gesellschaftliche, politische und medizinische, darunter auch kinder- und jugendpsychiatrische Aufmerksamkeit. Oftmals - aber durchaus nicht immer - sind mit der „verfrühten“"Mutterschaft Risikohäufungen verbunden, die Entwicklungsbenachteiligungen für Mütter und deren Kinder nach sich ziehen. Diese Benachteiligungen betreffen sowohl die somatische als auch die psychische Gesundheit und können die gesellschaftliche Teilhabe von Mutter und Kind einschränken. Wie internationale und nationale Studien zeigen, sind die Risiken vielgestaltig (Holub et al. 2007, Boardman 2006, Lee und Gramotnev 2006, Shaw et al. 2006, Figueiredo et al. 2005, Trautmann-Villalba et al. 2004, Ziegenhain et al. 2003, Moffit et al. 2002, Hoffert 2002, Jaffee et al. 2001, Whitman et al. 2001; Fergusson und Woodward 1999, Camp 1996) und betreffen biologische, psychische und soziale Faktoren, die überdies in komplexen „Teufelskreisen“ entwicklungshemmende Wechselwirkungen entfalten können.

\section{Biologische und soziale Risikokonstellationen}

Biologische Risiken: Die somatischen Risiken für Schwangerschaft und Perinatalperiode werden kontrovers diskutiert. Das Thema wird in der internationalen Literatur bereits seit mehreren Jahrzehnten behandelt, über die letzten Jahre lässt sich darüber hinaus eine Renaissance des Themas beobachten. Dieses Wiederaufleben hat mehrere Gründe. Erstens, das Risikokonzept wurde verfeinert; zweitens, die Messung abhängiger Variablen wurde revolutioniert (Fortschritte in der Neurophysiologie); drittens, Modelle der transaktionalen Wechselwirkung von Biologischem und Sozialem werden komplexer und werden in der Fachwelt zunehmend besser akzeptiert.

Zahlreiche Publikationen widmen sich auch heute noch solitär der Frage prä-, peri- und postnataler Komplikationen bei minderjährigen im Vergleich zu volljährigen Müttern. Auf der einen Seite finden sich Artikel, die vermehrte Komplikationsraten i.S. von Zervixinsuffizienzen, Frühgeburten, Hypotrophien und erhöhter Morbidität der Kinder minderjähriger Mütter beschreiben (Scholl et al. 1994, Perry et al. 1996, Hediger et al. 1997, Wallace 2001, Menacker et al. 2004, Birkeland et al. 2005, Goldenberg et al. 2005, Yilidrim et al. 2005). Yilidrim et al. (2005) beschreiben in ihrer nicht kontrollierten Untersuchung das niedrige Geburtsgewicht als häufigste Komplikation bei jungen Müttern, welches sie jedoch nicht mit biologischer Unreife, sondern mit mangelnden Vorsorgeuntersuchungen in Verbindung bringen. Auf der anderen Seite sind nach Coley und Chase-Lansdale (1998) in der Literatur Angaben zu erhöhten Komplikationsraten rückläufig. Diese Arbeitsgruppe bringt die Geburtskomplikationen minderjähriger Mütter eher mit schlechterer Vorsorge und Armut als mit dem Alter der Mutter in Verbindung. Die Mehrzahl der Ergebnisse spricht jedoch dafür, dass bei sorgfältiger Kontrolle anderer Faktoren 
keine erhöhten Raten biologischer Komplikationen vorliegen (Walcher et al. 1989, Plöckinger et al. 1996, Ambadekar et al. 1999, Hamada et al. 2004, Haerty 2004, Zeteroglu et al. 2005). Insbesondere ist hier die Arbeit von Mariotoni et al. (1998) hervorzuheben, in der bei sehr jungen Müttern kein erhöhtes Risiko für ein niedriges Geburtsgewicht festgestellt wurde, sobald die Daten für sozio-ökonomische Faktoren kontrolliert wurden.

Soziale Risiken: Auch die Wirkungen individueller und kontextueller Risiken werden in der Wissenschaft unterschiedlich aufgefasst. Fest steht, dass viele soziale Risiken mit Teenager-Schwangerschaften assoziiert sind, was sowohl die Verursachung als auch die Folgen betrifft. Häufig genannte Faktoren sind hier

- geringe Bildung,

- Arbeitslosigkeit,

- ungünstige Familienverhältnisse,

- Alleinerziehung,

- soziale Devianz oder

v sexuelle Promiskuität (s. Kap. 8.3).

Derartige soziale Risikokonstellationen wiederum sind nicht nur mit prä-, peri- und postnatalen Risiken biologischer Natur verbunden, sondern manifestieren sich häufig in individuellen Eigenschaften von Teenager-Müttern, die ihrerseits biologische und soziale Risiken für die Entwicklung von Mutter und Kind bedingen. In vielen Publikationen wird beispielsweise auf den (multiplen) Konsum legaler und illegaler Drogen bei sehr jungen Müttern aufmerksam gemacht (Kokotailo et al. 1994, Barnet et al. 1995, Gama et al. 2002, Kuchenbecker 2004, Gisselmann 2005).

Es bleibt jedoch anzumerken, dass es „den Typ“ der adoleszenten Mutter nicht zu geben scheint. Bereits 1987 stellten Furstenberg, Brooks-Gunn und Morgen für die USA fest, dass das Phänomen „frühe Mutterschaft" zuviel Variabilität aufweist, als dass sich einfache Wirkmechanismen für ihre Verursachung annehmen ließen, wie z. B. die Annahme, dass verfrühtes Sexualverhalten und supportive Partnerschaft einander ausschlössen. Offenbar gibt es viele Wege in die frühe Mutterschaft, von denen nicht alle dysfunktional sind.

Ein dysfunktionaler Mechanismus, der als individuelles Risiko für frühe Schwangerschaft zunehmend diskutiert wird, ist die psychische Erkrankung der Mutter. Offenbar sind psychisch kranke Mädchen und junge Frauen nicht nur gefährdeter, früh Mutter zu werden, sondern auch stärker in Gefahr, mit den nachfolgenden Erziehungsaufgaben überfordert zu sein. Insbesondere Persönlichkeitsstörungen sowie depressive und Angsterkrankungen werden dabei als mögliche Störungsbilder diskutiert (Maughan und Lindelow 1997, Fergusson und Woodward 1999, Ziegenhain et al. 2003, Moffit et al. 2002, Birkeland et al. 2005).

Erstaunlicherweise gibt es nach dem Wissen der Autoren jedoch keine Studien, die den Zusammenhang von $\mathrm{AD}(\mathrm{H}) \mathrm{S}$ und früher Mutterschaft untersuchen. Die vorliegende Arbeit soll beginnen, diese Lücke zu schließen. Sie dis- 
kutiert, inwieweit die Aufmerksamkeits-Hyperaktivitätsstörung (ADHS) am Zustandekommen früher Schwangerschaften und/oder an der Entstehung und Aufrechterhaltung nachfolgender Problemlagen und Fehlentwicklungen bei Mutter und Kind beteiligt sein könnte.

\subsection{Belege für den Zusammenhang von Teenager-Schwangerschaft und ADHS}

Die Idee eines Zusammenhanges von Teenager-Schwangerschaft und ADHS stammt zunächst aus unserer kindseitigen praktisch-klinischen Erfahrung. Eine Analyse unserer stationären Patientenklientel der Klinik für Psychiatrie, Neurologie, Psychosomatik und Psychotherapie des Kindes- und Jugendalters der Universität Rostock im Jahr 2003 zeigt, dass 5,5\% aller Patienten geboren wurden, als ihre Mütter $\leq 18$ Jahre alt waren bzw. 13,7\% als ihre Mütter $\leq 20$ Jahre alt waren. Verglichen mit dem oben angegebenen Bundesdurchschnitt lag die Prävalenz minderjähriger Mütter im Klinikklientel also etwa viermal höher als in der Crundgesamtheit. Unter den Kindern der unter 18 Jahre alten Mütter wurden 59 \% mit einer F9-Erstdiagnose nach ICD 10 geführt, von den Kindern der unter 20-jährigen Mütter etwa die Hälfte. Damit lag der genetischätiologische Verdacht nahe, dass einige dieser Mütter selbst eine $\mathrm{AD}(\mathrm{H}) \mathrm{S}$ oder eine andere psychische Störung aufweisen.

Dieser offensichtliche Zusammenhang hat jedoch bisher in der Fachliteratur kein Echo gefunden (s. Tab. 6 und 7).

Tab. 6 Ausgewählte Studien zu früher Mutterschaft

\begin{tabular}{|c|c|c|c|c|}
\hline Autor & Jahr & Land & Stichprobe & Studiendesign \\
\hline $\begin{array}{l}\text { Fergusson DM und } \\
\text { Woodward LJ }\end{array}$ & 1999 & NZ & $\begin{array}{l}\mathrm{N}=1.025 \\
\leq 20 \mathrm{I}\end{array}$ & Kinder 18 J. \\
\hline $\begin{array}{l}\text { Moffit TE and the } \\
\text { E-Risk Study Team }\end{array}$ & 2002 & UK & $\begin{array}{l}N=1.116 \\
50 \%<20 \mid\end{array}$ & $\begin{array}{l}\text { längsschnittliche Zwillings- } \\
\text { untersuchung }\end{array}$ \\
\hline Hillis SD et al. & 2004 & USA & $\begin{array}{l}N=9.159 \\
\geq 18 \mathrm{~J}\end{array}$ & $\begin{array}{l}\text { retrospektive Kohortenstudie } \\
\text { „Adverse Childhood Experiences“ }\end{array}$ \\
\hline Ziegenhain U et al. & 2003 & D & $N=29$ & Interventionsstudie \\
\hline Shaw M et al. & 2005 & AUS & $\begin{array}{l}N=460 \\
\leq 18\end{array}$ & $\begin{array}{l}\text { prospektiv, Mater University Study of } \\
\text { Pregnancy, U1 und } 14 \mathrm{~J} \text {. } \\
\text { ( } \mathrm{n}=4.800<18 \text { ).) }\end{array}$ \\
\hline $\begin{array}{l}\text { Lee } \mathrm{C} \text { und } \\
\text { Gramotev } \mathrm{H}\end{array}$ & 2006 & AUS & $\begin{array}{l}N=9.800 \\
18-23 \mid\end{array}$ & $\begin{array}{l}\text { prospektiv, „Australian longitudinal } \\
\text { study on women's health“ }\end{array}$ \\
\hline Holub CK et al. & 2007 & USA & $\begin{array}{l}N=154 \\
14-19 \mid\end{array}$ & $\begin{array}{l}\text { prospektiv vom } 3 \text {. Trimester bis } \\
16 \text { Monate alt }\end{array}$ \\
\hline
\end{tabular}


Tab. 7 Ausgewählte Studien zu ADHS bei Mädchen

\begin{tabular}{|c|c|c|c|c|}
\hline Autoren & Jahr & Land & Stichprobe & Studiendesign \\
\hline $\begin{array}{l}\text { Hinshaw SP } \\
\text { Lee SS und Hinshaw SP } \\
\text { Briscoe-Smith AM } \\
\text { und Hinshaw SP }\end{array}$ & $\begin{array}{l}2002 \\
2006 \\
2006\end{array}$ & USA & $\begin{array}{l}n=140 \\
6-12 J\end{array}$ & $\begin{array}{l}\mathrm{N}=88 \text { matched controls (Alter }+ \\
\text { Ethnie), Subkategorien } \mathrm{AD}(\mathrm{H}) \mathrm{S} \\
\text { gebildet }\end{array}$ \\
\hline $\begin{array}{l}\text { Rucklidge || und } \\
\text { Tannock R }\end{array}$ & 2001 & Kanada & $\begin{array}{l}n=24 \text { girls } \\
n=35 \text { boys } \\
13-16 \mid\end{array}$ & $\begin{array}{l}\text { männl. und wbl. Kontrollen mit } \\
\text { und ohne ADHS }\end{array}$ \\
\hline Biederman J et al. & 2008 & USA & $\begin{array}{l}n=140 \text { girls } \\
6-18 J .\end{array}$ & $\begin{array}{l}\text { prospektiv für } 5 \text { Jahre, wbl. Kontrollen, } \\
\text { Population aus Psych. und Pädiatrie }\end{array}$ \\
\hline Gaub M und Carlson CL & 1997 & (USA) & & Metaanalyse an 18 Studien \\
\hline
\end{tabular}

Unter den im Thesaurus der APA im März 2007 nachgeschlagenen Schlagwörtern „adolescent pregnancy/mother/father* attention deficit disorder/hyperactivity/psychiatric disorder" haben wir bei vollständiger kreuzweiser Verknüpfung in den Datenbanken MEDLINE, PsychInfo und Psyndex keine „Treffer gelandet". Lediglich bei der Google-Suche stießen wir auf eine Hördatei eines Interviews, in dem Cordula Neuhaus (Heilpädagogin und verhaltenstherapeutisch ausgewiesene Psychologin sowie Autorin einiger Sachbücher zu ADHS; 2006) aussagt, dass „Die Teenagerschwangerschaftsrate bei Mädchen mit ADHS bei $40 \%$ liegt. "Die Aussage wird nicht wissenschaftlich begründet, sondern geht auf persönliche und sonstige Erfahrungswerte zurück.

Die Gründe für das Fehlen einschlägiger Studien sehen wir erstens im erst kürzlich ausgeweiteten „ADS-Horizont“, womit die Störung nicht nur als lebenslanges, sondern zunehmend auch als weibliches Phänomen beschrieben wird, und zweitens in einer in der deutschen Forschung noch nicht stattgefundenen Hinwendung zur frühen Mutterschaft. Die Bearbeitung des Themas wäre jedoch durchaus lohnenswert, gerade dann, wenn es um das Thema ADHS geht.

Nach unserer Ansicht haben kontextuelle Faktoren wie Armut, ethnische Zugehörigkeit, Familienstruktur usw. nur einen begrenzten Erklärungswert, wenn sie ohne individuelle Voraussetzungen als Prädiktoren für Eintreten und Folgen früher Mutterschaft verwendet werden. Gerade unter Bedingungen relativer Versorgungsgleichheit, wie sie in Deutschland (noch) gegeben sind ${ }^{2}$, sollten sich individuelle Risiken wie Psychopathologie, genetische Dispositionen und körperliche Gesundheit relativ stärker auswirken als Kontextfaktoren, wie sie z. B. in den USA stärkere Wirkung entfalten (s. Tab. 8).

2 Dabei soll keinesfalls die in Deutschland immer weiter aufklaffende soziale Schere verharmlost werden, sondern darauf hingewiesen werden, dass in Deutschland die Varianz im Phänomen „frühe Mutterschaft" per se weniger aus sozialer Varianz erklärt werden kann als z. B. im Vergleich zu den USA. Allerdings ist zu beachten, dass mit der institutionellen Unterstützung für Kinder (Kindergeld, evtl. Ausbildungschancen) auch Anreize zur frühen Mutterschaft entstehen können, wie sie in den USA kaum denkbar sind. 
Tab. 8 Gegenüberstellung von Merkmalen früher Mutterschaft und $A D(H) S$ bei Mädchen

\begin{tabular}{|c|c|c|}
\hline Konstrukt & Frühe Mutterschaft & AD(H)S bei Mädchen \\
\hline \multicolumn{3}{|l|}{ MUTTER } \\
\hline Bildung & weniger gebildet & $\begin{array}{l}\text { niedriger IQ und schlechte } \\
\text { Schulergebnisse }\end{array}$ \\
\hline $\begin{array}{l}\text { Unterstützungs- } \\
\text { situation }\end{array}$ & $\begin{array}{l}\text { alleinerziehend, mangelhaft sozial } \\
\text { unterstützt, während der Kindheit } \\
\text { oft unsichere Bindung und/oder } \\
\text { Opfer von Missbrauch, } \\
\text { Vernachlässigung }\end{array}$ & $\begin{array}{l}\text { häufig Trennung und familiäre } \\
\text { Zerwürfnisse } \\
\text { (eigene und elterliche) }\end{array}$ \\
\hline psychische Probleme & $\begin{array}{l}\text { depressiv, geringes } \\
\text { Selbstwertgefühl }\end{array}$ & $\begin{array}{l}\text { komorbide affektive und } \\
\text { Angststörungen, Enuresis, } \\
\text { Ticstörung; geringes Selbstwert- } \\
\text { gefühl; Verhaltensprobleme, } \\
\text { Persönlichkeitsstörungen }\end{array}$ \\
\hline Drogenkonsum & legale und illegale Drogen & legale und illegale Drogen \\
\hline Sozialkompetenz & $\begin{array}{l}\text { weniger feinfühlig u. responsiv, } \\
\text { geben weniger Anregung }\end{array}$ & $\begin{array}{l}\text { Hyperaktivität, Impulsivität, } \\
\text { Aufmerksamkeitsschwäche, } \\
\text { ineffektive interpersonale } \\
\text { Wahrnehmung }\end{array}$ \\
\hline Kontrollüberzeugungen & nicht bekannt & $\begin{array}{l}\text { externale Kontrollüberzeugung, } \\
\text { maladaptive Attribuierung } \\
\text { negativer Ereignisse }\end{array}$ \\
\hline Sexualität & $\begin{array}{l}\text { riskantes Sexualverhalten, } \\
\text { frühe Schwangerschaft }\end{array}$ & riskantes Sexualverhalten \\
\hline
\end{tabular}

Für die These der „Komorbidität“ von ADHS und früher Mutterschaft spricht eine vergleichende Betrachtung (s. Tab. 8). Einige der hier für die ADHS benannten Merkmale treffen auch für andere psychische Problemlagen zu, wie z. B. Persönlichkeits- und Verhaltensstörungen. Aus unserer Sicht hat von allen in Frage kommenden Störungsbildern jedoch die ADHS für frühe Mutterschaft die größte Relevanz, da sie sich weitaus früher manifestiert und oft die primäre Beeinträchtigung bildet, aus der andere psychische Probleme folgen. Zudem weist die ADHS die größte Prävalenz aller kinder- und jugendpsychiatrischen Erkrankungen auf (siehe z. B. Kap. 1).

Von der ADHS ist mittlerweile bekannt, dass sie lebenslange Implikationen hat, einmal durch eine bemerkenswerte Persistenz ins Erwachsenenalter, andererseits als Anfangsproblem von Problemketten, innerhalb derer sich Person- und Unweltvariablen unentwirrbar miteinander verbinden. Damit darf angenommen werden, dass eine ADHS während der gesamten Zeit früher und späterer Elternschaft „wirkt“, d.h. am Zustandekommen einer Teenager- 
Schwangerschaft ebenso beteiligt ist wie an ihren Folgen. Aus diesem Grunde haben wir die folgende Diskussion nach Müttern und Kindern aufgeteilt und chronologisch geordnet. Wir beginnen mit der Verbindung von ADHS und früher Mutterschaft.

\section{Mutterseitige Risiken}

Die Ursachen früher Mutterschaft sind äußerst vielgestaltig, so dass hier nur ein kurzer Überblick zu möglichen Problembereichen gegeben werden soll, die für die Diskussion des Zusammenhangs von ADHS und Schwangerschaftsrisiko nützlich sind.

Inwieweit welche Gründe beim Zustandekommen einer Teenager-Schwangerschaft eine Rolle spielen, hängt sowohl von der Person der jungen Mutter als auch von den sie begleitenden Lebensumständen ab. Beide lassen sich nicht voneinander trennen und sollen in ihrer Wechselwirkung für den Lebensverlauf einer jungen Mutter im Folgenden beschrieben werden. Grundsätzlich lassen sich Kausalitäten für das Zustandekommen einer zu frühen Elternschaft bis in die Zeit vor der Geburt der Teenager-Mutter zurückverfolgen, z. B. zum Gebärverhalten der Großmutter oder zu Familiengeheimnissen wie transgenerationalen Inzestmustern. Der Einfachheit halber wird hier mit der Kindheit der Mutter begonnen.

Bereits Kindheit und Jugend der minderjährigen Mutter sind durch auffällige Merkmale gekennzeichnet. In der Literatur werden vor allem die folgenden Faktoren genannt:

- Herkunft aus zerrütteten Familien, aus Single-Parent-Haushalten (Laucht 1992a),

- niedriger eigener und elterlicher Bildungsstand (Alexander und Guyer 1993, Martin et al. 2005),

- schlechtere sozio-ökonomische Umstände (Figueiredo et al. 2005, Maynard 1996, Martin et al. 2005),

- häufiger Substanzmissbrauch (Seamark und Gray 1998, Kuchenbecker 2004, Figueiredo et al. 2005, Gisselmann 2005). Nach Kokotailo et al. (1994) konsumieren pränatal 35\% der jungen Mütter Alkohol und/oder Cannabis und ca. $50 \%$ rauchen Nikotin. Einer eigenen Untersuchung (Barchmann 2008) zufolge besteht ein signifikanter Unterschied im Nikotinkonsum zwischen unter 18-jährigen und 18- bis 35-jährigen Schwangeren. In der erstgenannten Gruppe ist der Anteil an Raucherinnen mehr als doppelt so hoch.

- Hohe Prävalenzen für Misshandlung und sexuellen Missbrauch (Maughan und Lindelow 1997, Taylor et al. 1999, Saewyc et al. 2004, Harner 2005) werden genannt. Taylor et al. (1999) zeigen eine Verbindung zwischen Bildung und Alter des gewählten Geschlechtspartners auf: Je niedriger der Bildungsstand des Mädchens ist, desto größer wird die Altersdifferenz zum Partner. Graham (1991) bemerkt, dass Teenager-Schwan- 
gerschaften zu einem hohen Prozentsatz aus inzestuösen Vater-TochterBeziehungen und weniger aus kurzen promiskuösen Beziehungen zu gleichaltrigen oder älteren Partnern entstammen. Saewyc et al. (2004) geben an, dass von sexuell missbrauchten Frauen 13 bis $26 \%$ als Teenager schwanger wurden.

Bereits zu diesem frühen Zeitpunkt ist es wahrscheinlich, dass eine ADHS in differenzierter Weise mit den genannten Risiken zusammenwirkt. Was sexuellen Missbrauch angeht, so ist bekannt, dass gerade Kinder mit Aufmerksamkeitsproblemen, die soziale Reize nur unzureichend dekodieren, häufiger Opfer werden. Mädchen mit ADHS werden nach einer Untersuchung von BriscoeSmith und Hinshaw (2006) etwa dreimal häufiger missbraucht als gesunde Mädchen (14,3\% vs. 4,5\%), wobei unter den Opfern der gemischte Typ der ADHS weit häufiger vorkam als der rein unaufmerksame Typ. Die Wahl der Sexualpartner dürfte durch Impulsivität und die ineffektivere Verarbeitung sozialer Reize schon früh negativ beeinflusst werden. Es lässt sich annehmen, dass impulsive Mädchen weniger wählerisch sind und geringere Vorkehrungen gegen ungewollte Schwangerschaften treffen. Gleichzeitig darf angenommen werden, dass die belasteten Eltern-Kind-Beziehungen zu einem relativ frühen Abbruch des elterlichen Monitorings führen, womit sich für die Mädchen weitere Gelegenheiten für frühe Schwangerschaften ergeben. Für Jungen sind derartige Mechanismen in einer Studie von Flory et al. (2006) belegt worden, nach der eine ADHS in der Kindheit frühere sexuelle Aktivität, eine höhere Zahl an Sexualpartnern und mehr Gelegenheitssex vorhersagt. Auch wenn die mit einer ADHS verknüpften Verhaltensstörungen zum riskanten Sexualverhalten beitragen, so ist der o. g. Untersuchung zufolge dennoch auch ein davon unabhängiger Anteil der Kernsymptome der ADHS ursächlich beteiligt. Nach Monuteaux et al. (2007) ist ADHS auch bei Mädchen ein Risikofaktor zur Ausbildung eines gestörten Sozialverhaltens und damit assoziierter Bildungsbenachteiligungen, erhöhter psychiatrischer Morbidität und auffälligen Sexualverhaltens.

Weiter ist bekannt, dass Kinder mit einer ADHS deutlich geringere Ressourcen in der Austragung von Familienkonflikten, wie sie in zerrütteten Familien wahrscheinlicher sind, haben (Kaeppler 2005). Kinder mit einer ADHS neigen zu schnellerem Einstieg in den Drogenkonsum, insbesondere wenn die Störung unbehandelt bleibt und mit einer Störung des Sozialverhaltens vergesellschaftet ist (Barkley et al. 2004). Davon ist insbesondere der hyperaktiv-impulsive Subtypus betroffen, bei dem nach Elkins et al. (2007) auch ohne Vorhandensein einer Störung des Sozialverhaltens ein klarer Zusammenhang zu Drogenmissbrauch besteht. Effekte niedrigeren Bildungsstandes wiederum, wie z. B. extensiver Medienkonsum (Moessle et al. 2007), werden durch ADHS verstärkt, da die betroffenen Kinder ihre begrenzte Aufmerksamkeitsleistung auf bildungsirrelevante Verstärker verwenden.

Cründe verfrühter Schwangerschaften werden auch in Einstellungen zur Sexualität (Friedrich und Remberg 2005), adoleszenten Autonomiebestrebun- 
gen (Bluestein 1994, Hurlbut 1997, Ziegenhain et al. 2003), der Familienstruktur (transgenerationale Weitergabe) sowie in der sozialen Mobilität (Lawrence 1993) gesehen.

Einstellungen zur Sexualität: Nach den Daten einer BZgA Studie (2001) haben $10 \%$ der unter 14-Jährigen Erfahrungen mit Geschlechtsverkehr, bei den 14-17-Jährigen sind es $30 \%$, bei den über 17-Jährigen sind es zwei Drittel aller Befragten. Dabei wird zum Verhütungsverhalten aus der gleichen Untersuchung bekannt, dass zwei Drittel der Teenager beim „ersten Mal“ nicht verhüten. Es gilt, je früher der Geschlechtsverkehr stattfindet, desto unvorbereiteter sind die Mädchen (BZgA 2001). Einflussfaktoren sind hierbei Bildungsstand, religiöse Einstellung, Wissen der Eltern, Vertrauen in die Mutter, Sexualerziehung in der Schule und Kommunikation im Elternhaus bzw. mit dem Partner (Remberg und Weiser 2003). Die These vom „unaufgeklärten Teenager“ wird allerdings kontrovers diskutiert. In jedem Fall erklärt schlechtere Aufklärung über Sexualität und Kontrazeption nicht hinreichend das Phänomen sehr früher Schwangerschaft (Häußler-Sczepan 2005).

Entwicklungspsychologische Aspekte: Im Zuge von Autonomiebestreben/Trennung von Eltern, der Übernahme der Frauen - und Erwachsenenrolle und der Selbstverwirklichung (Osthoff 200o) kann es im Sinne psychodynamischer Ansätze zur Ausprägung eines so genannten „pathologischen“ Kinderwunsches kommen. Ein eigenes Kind wird hierbei zu einem Mittel der Konfliktlösung auf der Suche nach familiärer/mütterlicher Geborgenheit oder nach Liebe und Wärme (Garst 2003). Manchmal soll auch die Bindung zum Partner über ein gemeinsames Kind intensiviert oder überhaupt erst hergestellt werden (Wanzeck-Sielert 2002).

Familienstruktur- und Sozialhypothesen gehen sowohl von einer transgenerationalen Weitergabe (von der Mutter zur Tochter, Kirchengast 2002) und Nachahmung sozialer Entwicklungsmodelle im Bekanntenkreis als auch von der Erfüllung eigener Perspektiven bei schlechten Ausbildungsmöglichkeiten sowie der Suche nach gesellschaftlicher Anerkennung und einer gewissen finanziellen Absicherung aus (14. Shell Jugendstudie, Hurrelmann und Albert 2002).

Für die Zeit von Schwangerschaft und bei Geburt wird für Teenagermütter im Vergleich zu älteren Müttern das Auftreten einer verspäteten und schlechteren Vorsorge (Figueirido 2006, Kaiser und Hays 2005, Goonewardene und Deeyagaha 2005, Gilbert et al. 2004, Gama et al. 2002, Coley und Chase-Lansdale 1998), eine häufigere Einweisung per Notfall (Fracassi 2008) und ein erhöhter Nikotinkonsum (Barchmann 2008, Viertler 2008, Gilmore et al. 2006, Archie et al. 1997, Barnet et al. 1995, Kokotailo 1994) beschrieben. Allerdings werden das gehäufte Vorkommen von Schwangerschafts- und Geburtskomplikationen einerseits (Keskinoglu et al. 2007, Malamitsi-Puchner und Boutsikou 2006) und das somatische Outcome bei den Neugeborenen andererseits (Markovitz et al. 2005, Gilbert et al. 2004, Mathews et al. 2003) gegensätzlich diskutiert. Danach finden sich bei jungen Müttern eventuell Häufungen von biologischen Risiken, wie sie als Ursache für problematische Frühentwicklungen, nicht aber 
für lebenslange Folgen ausgemacht wurden (Meyer-Probst und Teichmann 1984, Laucht et al. 1992, Sameroff 2006).

Auch für Schwangerschaft und Geburt müssen enge Interaktionen von ADHS und Teenager-Risiken angenommen werden. So ist bekannt, dass ADHSBetroffene schneller zur Zigarette greifen, schon um kurzzeitig ihre Konzentrationsleistungen zu erhöhen (Bekker et al. 2005, Fuemmeler et al. 2007, Collins und McClernon 2007, Potter und Newhouse 2004) und damit anfälliger für Fehlkonditionierungen im Zusammenhang mit Nikotin sind (Reichel et al. 2007). Weiterhin werden eher impulsungesteuerte Mädchen umso geringere Chancen haben, mit dem Rauchen während der Schwangerschaft aufzuhören, je jünger sie sind. Im Allgemeinen ist bekannt, dass signifikant mehr jüngere als ältere Schwangere rauchen (Barchmann 2008, Seamark und Gray 1998). Damit sind Teenager-Mütter gefährdeter, was nikotinbezogene Risiken für Mutter und Kind angeht. Das beginnt bei möglicherweise intrauteriner Unterversorgung des Embryos und der Wirkung des Nikotins als Zellgift (Wakschlag et al. 2002); spannt sich weiter über kindliche Belastungen durch Passivrauchen und reicht bis hin zu einem schlechteren Verlauf bei ADHS. Nikotinkonsum der Mutter während der Schwangerschaft wiederum hat erwiesenermaßen einen Einfluss auf spätere ADHS-Symptome des Kindes (Button et al. 2005, Laucht und Schmidt 2004), womit hier Summationseffekte von genetischer Prädisposition und Nikotineinfluss wahrscheinlich werden.

Weiterhin ist wahrscheinlich, dass zum einen Mädchen mit ADHS weniger medizinische Behandlung erfahren als Jungen mit ADHS (Derks et al. 2007), wobei noch einmal Mädchen gefährdeter sind, die aus ungünstigen sozialen Verhältnissen kommen. Mädchen fallen als „ADHS-Kinder“ deshalb weniger auf, weil sich ihre Störung „,rollentypisch“ manifestiert - eher als Aufmerksamkeits- denn als Impulsivitätsproblematik.

Zu den postpartalen Merkmalen adoleszenter Mütter ist bekannt, dass sie eine höhere psychiatrische Morbidität (Maughan und Lindelow 1997, Fergusson und Woodward 1999, Ziegenhain et al. 2003) aufweisen. Am häufigsten wird in der Literatur das Auftreten von internalisierenden Störungen beschrieben. Adoleszente Mütter sind nach Literaturlage stärker von postpartalen Depressionen bedroht (Black 2002, Moffit et al. 2002, Freitas 2002, Birkeland et al. 2005). Eine - sich bei Teenagern stärker als bei älteren Müttern auswirkende - enge Korrelation zwischen erhöhtem psychischen Stress und postpartaler Depression wird von Barnet et al. (1996) erwähnt. Dieser Zusammenhang kann vermutlich mit dem Fehlen emotionaler und sozialer Unterstützung erklärt werden (Barnet et al. 1996, Coley und Chase-Lansdale 1998). Auch Angststörungen treten bei jungen Müttern gehäuft auf (Piyasil 1998, Fergusson und Woodward 1999). Einen unmittelbaren Einfluss auf die Anpassungsfähigkeit und Entwicklung des Kindes hat die durch die geringere mütterliche Responsivität und Feinfühligkeit der Mutter gestörte Interaktion (Laucht et al. 1992a, Ziegenhain et al. 2003, Trautmann-Villalba et al. 2004). Weiterhin können die Risiken, die das Zustandekommen der ersten Schwangerschaft begleiten, für weitere Schwangerschaften verantwortlich sein. So ist über die weitere Entwicklung 
junger Mütter bekannt, dass sie mehr Kinder bekommen und dennoch häufiger allein erziehend bleiben (Alexander und Guyer 1993, Moffit et al. 2002, Figueiredo 2005). Zudem ist auch für Deutschland bekannt, dass immer mehr Kinder und Jugendliche - insbesondere in den neuen Bundesländern - unter der Armutsgrenze (Orientiert sich am Netto-Haushaltseinkommen der Familie, wenn dieser unterhalb von $50 \%$ - also des Medians - aller deutschen Haushalte liegt, dann wird von relativer Armut in der Familie gesprochen.) leben. Etwa $11 \%$ aller Kinder unter 18 Jahren sind von relativer Armut in Deutschland betroffen - vergleichsweise sind dies $21 \%$ in Mecklenburg-Vorpommern, $7 \%$ in Bayern und 4-5\% in Skandinavien (Bertram 2006). Während 2003 die Arbeitslosenquote für Deutschland bei 10, $5 \%$ lag, waren in den neuen Bundesländern $19,6 \%$ der Frauen (9,9\% in der BRD) und 16,1\% junger Menschen unter 26 Jahren arbeitslos (9,9\% in der BRD; Bundesagentur für Arbeit 2004). Nach Bertram (2006) führt nicht die ökonomische Deprivation allein, sondern ihre Einbettung in die Kumulation einzelner Dimensionen zur Benachteiligung. Hier schließt sich der Kreis, indem erlebte Perspektivlosigkeit eine Motivation für erneuten Kinderwunsch werden kann, um darüber eine gewisse finanzielle Absicherung und gesellschaftliche Anerkennung zu erfahren (siehe oben). Hat eine Mutter zum Zeitpunkt der Schwangerschaft bereits eine Ausbildung begonnen, so ist sie mit anderen Problemen konfrontiert. Billari und Dimiter (2006) zeigten in einer Erhebung zu Bildungsverläufen bei Müttern in Westeuropa, dass die Wahrscheinlichkeit eines vorzeitigen Ausbildungsabbruches für junge Mütter in z. B. Deutschland und Österreich höher liegt als in Nordund Südeuropa. Dieser Unterschied lässt sich durch zwei mögliche Ursachen erklären. Einerseits wird die Vereinbarkeit von Muttersein und Ausbildung durch deutsche Familienpolitik im Vergleich zu Nordeuropa nicht ausreichend gefördert. Auf der anderen Seite scheinen deutsche Familien den in Südeuropa vorherrschenden familialen Zusammenhalt weniger zu bieten. Bleibt eine Mutter in Deutschland alleinerziehend, so ist der (Wieder)-Einstieg in Ausbildung oder Beruf erschwert. Zwischen dem Alleinerziehungsstatus, dem vergleichsweise niedrigeren Bildungsniveau und der Langzeitarbeitslosigkeit/ Abhängigkeit von Sozialhilfe (Jaffe 20o1) bestehen demnach direkte Wechselwirkungen, die durch das Vorhandensein einer psychischen Erkrankung wie bspw. eine ADHS noch zusätzlich negativ beeinflusst werden.

Die belastete Mutter hat größere Schwierigkeiten bei der Inanspruchnahme kompetenter Hilfe für ihr Kind, beim Aufstellen/Einhalten klar strukturierter Erziehungsregeln, bei der Inanspruchnahme von Hilfe kindzentrierter Institutionen (z. B. Kindergarten, Jugendamt), bei der Mobilisierung externer Ressourcen und bei der Trennung eigener und kindzentrierter Probleme. Das Beobachten und Einschätzen kindlichen Verhaltens fällt ADHS-Müttern (Murray 20o6) schwerer, womit sie weniger in der Lage sind, ihre Maßnahmen situationsadäquat zu gestalten.

Für alle diese mutterseitigen Risiken gilt, dass sie entweder Ausdruck (Folge) einer ADHS der Teenager-Mutter sein können, oder aber dass sie durch eine vorliegende ADHS verstärkt werden. Andersherum lässt sich vermuten, dass 
eine vorliegende ADHS die Resilienz, also die Widerstandskraft, gegenüber den aufgezählten adversen Umwelt- und Personbedingungen mindert. Wenn eine junge Mutter beispielsweise Probleme hat, ihre eigenen Probleme von denen ihres Kindes zu trennen, in dem sie z. B. ihr Kind für die Unordnung der Wohnung verantwortlich macht, dann dürfte ihr dies umso schwerer fallen, je weniger sie selbst auf Grund einer ADHS Ordnung und Struktur halten kann. Die Rückmeldungen dem Kind gegenüber sind bei vorliegender ADHS eher impulsiv, überschießend und inadäquat, womit das Ausgangsproblem, in diesem Fall die fehlende persönliche Ordnung, wiederum eher verstärkt als gemindert wird.

\section{Kindseitige Risiken}

Gerade Teenager-Schwangerschaften verdeutlichen, wie eng Eltern- und Kindproblematiken in der Entstehung psychischer Krankheiten miteinander verwoben sind. Mutterseitige Risiken wirken unmittelbar auch kindseitig, solange Mutter und Kind gemeinsam leben.

Kindseitige Folgen früher Elternschaft können all jene sein, die allgemein für Risikomütter gelten. Insbesondere, wenn die Überforderung durch zu frühe Mutterschaft und ADHS zustande kommt, kann es schon sehr früh zu kindlichen Regulationsstörungen, wie sie aus der Literatur bekannt sind, kommen. Dabei handelt es sich um Schlafstörungen, Fütterstörungen und exzessives Schreien (Deutsche Gesellschaft für Kinder- und Jugendpsychiatrie und Psychotherapie 2007a). Bei gleichzeitig vorliegender familiärer psychopathologischer Belastung könnten sie als ein Hinweis auf eine ADHS oder andere psychische Anpassungsprobleme gewertet werden, also entweder als Frühsymptom oder als unspezifischer Vorbote späterer Problematiken. Die Mannheimer Risikokinderstudie belegt, dass ein signifikanter Zusammenhang von multiplen Regulationsstörungen und späterer ADHS in allen Altersstufen besteht. Bei Vorliegen mehrerer negativer psychosozialer Umstände ist von additiven Effekten und negativen Interaktionen auszugehen (Laucht und Schmidt 2004). Dabei soll gleichzeitig erwähnt werden, dass das „desregulierte“ Kind eine Reaktion auf die weniger responsive, depressive oder impulsive, schwer soziale Reize decodierende junge Mutter (mit eigener psychopathologischer Belastung, siehe oben) verstanden wird, die ihrerseits ohnehin schon mehr Probleme in der Organisation ihres Alltags hat. Hier wird wiederum die Aufsummierung, dynamische Wechselwirkung und negative Verstärkung widriger psycho-sozialer Umstände deutlich.

Die Interaktion von Mutter und Kind ist geprägt von (Moffit et al. 2002, Ziegenhain et al. 2003):

- weniger Aktivitäten/reizärmerer Umgebung,

- fehlender elterlicher Erziehungsübereinstimmung,

- mehr körperlicher Disziplinierung und

- weniger mütterlicher Wärme/mangelnder Feinfühligkeit. 
Dabei führen mangelnde Anregung und Förderung zu Verzögerung der kognitiven Entwicklung sowie Ablehnung und emotionale Vernachlässigung zu Verhaltensauffälligkeiten. Mit der weiteren Entwicklung des Kindes verlieren biologische Variablen an Einfluss, während sich psychosoziale umso stärker auswirken (Laucht 1992, Meyer-Probst und Teichmann 1984).

Folgen minderjähriger Mutterschaft für das Kind (Ziegenhain et al. 2003; Moffit et al. 2002, Jaffe 2001, Fergusson und Woodward 1999) finden sich häufig in folgenden Problematiken:

- Trennung von den Müttern (siehe auch Kuchenbecker 2004) und Leben in Pflegefamilien,

- Bindungsstörungen (siehe auch Coley und Chase-Lansdale 1998),

- Krankheiten und Unfällen (siehe auch Shaw et al. 2006),

- gefährdete kognitive und sprachliche Entwicklung, verbunden mit schlechter Schulbildung (siehe auch Shaw et al. 2006),

- Missbrauch/Misshandlung/Vernachlässigung

(siehe auch Maynard 1996),

- Drogenmissbrauch und

- aggressive Verhaltensauffälligkeiten/ADHS/Impulskontrollstörung/ Kriminalität bei Jungen (siehe auch Nagin et al. 1997, Nagin und Tremblay 2001, Hofferth und Reid 2002, Trautmann-Villalba et al. 2004).

In Outcome-Studien konnte gezeigt werden, dass das Vorliegen einer Teenager-Mutterschaft und eines niedrigen mütterlichen Bildungsniveaus Prädiktoren für die Persistenz von ADHS und Störungen des Sozialverhaltens über die Kindheit hinaus sind. In dieser Konstellation erhöht sich damit die Gefahr zur Entwicklung delinquenter und krimineller Verhaltensweisen (Morash 1989, Nagin et al.1997, 2001).

Nach Meyer-Probst und Teichmann (1984), Laucht (1992) und Coley und Chase-Lansdale (1998) sind ältere Kinder dabei von psycho-sozialen Entwicklungsdefiziten noch stärker betroffen als jüngere. Der Grund liegt darin, dass sie etwaigen Entwicklungsrisiken, wie z. B. nicht-responsiven Müttern, länger ausgesetzt waren als Kleinkinder, deren Entwicklung stärker durch biologische Reifungsprozesse beeinflusst wird (Meyer-Probst und Teichmann 1984; Sameroff 2006). Die $\mathrm{AD}(\mathrm{H}) \mathrm{S}$ wiederum, welche eine relativ hohe Persistenzrate von $60 \%$ hat, könnte wiederum dafür verantwortlich sein, dass negative Umweltbedingungen - wie z. B. die Alleinerziehung durch die Mutter relativ konstant bleiben, da die Mutter, bedingt durch ihre verringerte soziale Aufmerksamkeit - geringere Chancen auf gesunde Partnerschaft und die Beendigung der Alleinerziehung hat.

Daneben wird für Mädchen frühere sexuelle Aktivität mit der Folge früher Schwangerschaft beschrieben, wobei ca. ein Drittel dieser Mädchen selbst wieder Teenager-Mütter werden (Mc Cue Horwitz et al. 1991). Als Grund dafür wird von den Autoren vor allem emotionale Depravation in Form eines frühen Verlustes wichtiger Bezugspersonen und mütterlicher Depressivität diskutiert. Wir fragen uns, inwieweit auch das Vorliegen einer eigenen psychischen 
Störung diese Mädchen kennzeichnet, die ihrerseits wiederum frühzeitig Mütter werden.

Insgesamt muss davon ausgegangen werden, dass mutter- und kindseitige Risiken dazu neigen, sich gegenseitig in ihrer entwicklungsgefährdenden Wirkung zu verstärken. Wenn junge Mütter an einer ADHS leiden und dazu noch Kinder mit derselben Problematik bekommen bzw. „produzieren“, sind Mutter-Kind-Interaktionen schnell in Gefahr, sich zu lebenslangen Risikoschleifen „auszuwachsen“, die nur schwer zu durchbrechen sind und der Behandlung bedürfen.

\subsection{Folgerungen für Forschung und Praxis}

Zunächst kann aus den hier zusammengetragenen Ergebnissen geschlussfolgert werden, dass ADHS und frühe Mutterschaft ein Themenfeld aufspannen, das der weitergehenden intensiven Forschung bedarf. Bisher ist zu wenig über ADHS bei Mädchen bekannt, als dass sich sagen ließe, inwieweit diese relativ häufige Erkrankung am Zustandekommen problematischer Entwicklung in der Eltern- und der Kindergeneration beteiligt ist. $\mathrm{Zu}$ frühe Mutterschaft ist nach den bruchstückhaft vorliegenden Ergebnissen ein guter Kandidatenmechanismus, um die Persistenz psychiatrischer Problematiken in Familien zu erklären.

Hier sollen einige Forderungen aufgelistet werden, die sich nach unserer Ansicht für die Forschung und Praxis ergeben.

\section{ADHS in der Forschung zur frühen Mutterschaft}

Den Autoren ist bewusst, dass die folgenden Überlegungen Maximalanforderungen darstellen, die überdies noch nicht vollständig sind. Dennoch sollen sie hier aufgelistet werden, auch wenn wir uns im Klaren sind, dass Studien, die alle diese Forderungen erfüllen, einen hohen finanziellen Aufwand erfordern. Auf der anderen Seite darf man sich von Studien zu derartigen Hochrisikogruppen weit reichende Erkenntnisse für Prävention, Praxis und Intervention erhoffen, so dass Untersuchungen dieser Art uns allemal gerechtfertigt erscheinen, zumal der erwartete volkswirtschaftliche Nutzen die Kosten früher Interventionen weit überstiege. Im Detail sollte von wissenschaftlichen Studien Folgendes gefordert werden:

1. Die bisher in Deutschland gelaufenen Studien zur Beschreibung früher Mutterschaft sollten unbedingt durch Studien zum Zustandekommen und den Effekten von Teenager-Mutterschaft ergänzt werden.

2. Da es den „Typ der adoleszenten Mutter“ nicht zu geben scheint, sollten Studien zu diesem Thema groß genug angelegt sein, um nicht nur problematische Teenager-Mutterschaft zu beschreiben, sondern auch resiliente Entwicklungen. 
3. Aufmerksamkeits-Hyperaktivitätsstörungen sollten in derartigen Analysen Bestandteil des Untersuchungsmodells sein, denn sie haben vermutlich hohen Erklärungswert für problematische Verläufe.

4. Ursachen und Effekte sind nicht entweder an die Eltern- oder die Kindergeneration gebunden, sondern stehen lebenslang in einem wechselseitigen Zusammenhang. Aus diesem Grund sollten Erhebungen, die sich mit der Problematik befassen, wenigstens zwei Generationen untersuchen. Sowohl bei den Eltern ${ }^{3}$ als auch bei den Kindern sollten die Symptomatiken gemessen werden, möglichst nach den Leitlinien für ADHS-Diagnostik folgend (Deutsche Gesellschaft für Kinder- und Jugendpsychiatrie und Psychotherapie 2007 b, Ebert et al. 2003).

5. Von den Ursachen und Effekten muss angenommen werden, dass sie zeitgebunden auftreten, weshalb die zu planenden Studien längsschnittlich angelegt sein sollten und möglichst vor der Transition zur Elternschaft beginnen sollten.

6. Es kann davon ausgegangen werden, dass die beschriebenen Ursachen und Effekte nicht direkter Natur sind, sondern entweder nur in bestimmten Risikokonstellationen auftreten (Moderatormodell) oder aber durch andere Faktoren überhaupt erst vermittelt werden (Mediatormodell). Beispielsweise muss angenommen werden, dass frühe Mutterschaft nicht per se ein Entwicklungsrisiko darstellt. Oft werden junge Mütter mit älteren Müttern verglichen, ohne dass die Randbedingungen in beiden Cruppen parallelisiert werden. In einer Studie unserer Arbeitsgruppe, deren abhängige Variablen biologische Kindparameter zum Zeitpunkt der Geburt waren (Fracassi 2008), gelang es beispielsweise mit hohem Aufwand, junge und volljährige Mütter in einem matched-pair-Design zu vergleichen. Nachdem eine Gruppe von 100 minderjährigen Müttern einer Cruppe volljähriger Mütter $(n=100)$ gegenübergestellt wurde, die auf den Dimensionen Partnerstatus, Nikotinkonsum und Bildungsaspiration gleich belastet waren, ergaben sich keine Unterschiede mehr in Bezug auf Geburtsgewicht, Frühgeburtlichkeit und andere bekannte biologische Risiken.

7. Studien zum Zusammenhang von ADHS und Teenager-Schwangerschaft sollten spezifische Hypothesen prüfen. Insbesondere die Untertypen der ADHS sind möglicherweise mit verschiedenen Mediatoren gekoppelt, die die Entwicklung von Mutter und Kind beeinträchtigen. Beispielsweise darf von den eher aufmerksamkeitsgestörten Mädchen angenommen werden, dass sie schlechter soziale Reize dekodieren und darum häufiger viktimisiert werden, während eher impulsive Mädchen stärker zu riskantem Sexualverhalten und häufigen Partnerwechseln neigen dürften. Mädchen des gemischten Typs sollten ein höheres Risiko haben, externalisierende Verhaltensweisen zu entwickeln, womit sie vermutlich strafender/restriktiver gegenüber den eigenen Kindern sind.

3 Natürlich sind fast alle Problematiken die durch ADHS bei jungen Müttern entstehen, auch auf die Väter übertragbar. Väter sollten, wenn möglich, in die Studien einbezogen werden. 
8. Studien zum Zusammenhang von ADHS und Teenager-Schwangerschaft sollten auch interventiv sein. Es steht zu fragen, ob beispielsweise die Gabe von Methylphenidat auch Effekte auf Partnerwahl- und Sexualverhalten, genauso wie auf das Interaktionsverhalten nach der Geburt des Kindes hat. Bereits laufende Studien zu psychotherapeutischen Interventionen bei jungen Müttern, zu Trainings etc. sollten eventuell vorhandene Aufmerksamkeits- und Hyperaktivitätsstörungen zumindest berücksichtigen.

9. Untersuchungen zum Zusammenspiel von ADHS, früher Mutterschaft und nachfolgendem Erziehungsverhalten müssen multimodal erfolgen. Es darf angenommen werden, dass starke Entwicklungsrisiken wie sexueller Missbrauch oder vernachlässigendes Erziehungsverhalten nicht einfach mit Fragebögen „abgefragt“ werden können. Die erhöhte Wahrscheinlichkeit von traumatisierenden Familiengeheimnissen verlangt nach einer Kombination von narrativen und standardisierten Befragungen.

10. Frühe Mutterschaft sollte nicht - auch nicht im Zusammenhang mit ADHS - per se als Entwicklungsrisiko modelliert werden. In den Untersuchungen sollten möglichst nicht nur Defizitmodelle getestet werden, sondern auch protektive oder Resilienzfaktoren. Ein „zu frühes“ Kind kann unter Umständen auch Halt und Kraft für die junge Mutter bedeuten.

11. Die Wirkung frühe Mutterschaft ist stark abhängig von den Werturteilen, auf die sie trifft. Entsprechende Studien sollten deshalb immer den Kontext berücksichtigen, in dem ADHS und frühe Mutterschaft untersucht werden. Beispielsweise kann vorausgesetzt werden, dass in Regionen wie dem säkularen Mecklenburg-Vorpommern, wo die Großmütter ihre ersten Kinder durchschnittlich noch mit 20 Jahren bekamen, und in denen überdies für wenig gebildete Mädchen kaum Integrationsmöglichkeiten in den ersten Arbeitsmarkt bestehen, frühe Kinder eine andere Bedeutung haben als in anderen Ethnien Deutschlands, seien sie katholisch oder türkisch.

\section{ADHS und frühe Mutterschaft in der Praxis}

Junge Mütter werden, wenn sie nicht vorher selbst auffällig waren, zuerst beim Gynäkologen, Pädiater/Hausarzt oder der Hebamme vorstellig. Bereits hier sollte die Möglichkeit einer komorbiden ADHS berücksichtigt werden. Wie aus der Zusammenschau der bisherigen Ergebnisse ersichtlich wird, verstärkt eine ADHS die ohnehin mit früher Mutterschaft verbundenen Entwicklungsrisiken. Grundsätzlich sollte früh darauf hin gearbeitet werden, die Folgeprobleme einer ADHS bei früher Schwangerschaft so gering wie möglich zu halten. Wie aus den einschlägigen, anfangs zitierten Studien bekannt, geht es hierbei zuerst um die Herstellung eines vertrauensvollen Verhältnisses zur jungen Schwangeren, nicht allein um die Vermittlung in existierende Programme. Insbesondere Hebammen sind durch die größere Nähe zur jungen Mutter prädestiniert, erst dann beratend zu intervenieren, nachdem die jungen Mütter Vertrauen zu ihnen gefasst haben. Gegebenenfalls bedarf es hier intensiver 
Intervention, um entwicklungsgefährdende Faktoren zu minimieren. Dazu gehören effektive Rauchentwöhnungen ebenso wie Mobilisierungen sozialer und anderer Ressourcen schon während der Schwangerschaft, darunter auch die Übernahme in Beratungs- oder Therapieprogramme (Ziegenhain 2007).

Hinsichtlich einer pharmakologischen Behandlung mit Methylphenidat $(\mathrm{MPH})$ wird der Fachinformation entnommen, dass die Sicherheit beim Gebrauch während der Schwangerschaft nicht nachgewiesen wurde und kaum Untersuchungen dazu vorliegen (siehe auch Humphreys et al. 2007). Während der Schwangerschaft wird daher von einer MPH-Behandlung eher abgeraten. Bei einer ungefähr 10o-fachen maximalen Tagesdosis in mg/kg, die beim Menschen empfohlen wird, wurde beim Kaninchen eine teratogene Wirkung festgestellt. Nach Humphreys et al. 2007 soll es beim Menschen bei auch nicht sachgerechter Anwendung zwar zu keinen Malformationen kommen, aber dennoch können (andere) Schädigungen nicht ausgeschlossen werden. Da nicht bekannt ist, ob MPH in die Muttermilch übertritt, sollte als Vorsichtsmassnahme stillenden Müttern empfohlen werden, es nicht einzunehmen (siehe Fachinformation).

Im weiteren Verlauf sind auch Pädiater angehalten, die Problematik einer ADHS bei jungen Müttern ins Kalkül zu ziehen. Sollten sich während der Vorsorge- und Entwicklungsuntersuchungen für Kinder (U1 - U9 bzw. U11, J1, J2) Hinweise auf eine vorliegende Störung bei der Mutter (Warteraumverhalten!, Verhalten bei Terminabsprachen und während der Untersuchung) ergeben, so sollte die Mutter zur Diagnostik und Therapie bewegt werden, selbstverständlich wiederum nur, nachdem ihr Vertrauen gewonnen wurde. Möglicherweise kann so späteren Fehlentwicklungen bei Mutter und Kind vorgebeugt werden. Aktuelle Zahlen belegen, dass die Teilnahme an den Früherkennungsuntersuchungen mit den Jahren, d. h. vom 2. Lebensjahr bis zum Vorschulalter, sinkt. Gerade Kinder aus sozial schwachen Elternhäusern nehmen weniger an der U7 bis U 9 teil. Das führt dazu, dass z. B. Entwicklungsstörungen vor der Einschulung häufig unerkannt bleiben und damit auch nicht rechtzeitig behandelt werden können (BZgA 2008). In diesem Zusammenhang begrüßen wir die Schritte hin zu einem verpflichtenden Status der Vorsorgeuntersuchungen. Bundesweit engagieren sich lokale Netzwerke für eine breitere Aufklärung zu den Vorsorgeuntersuchungen. Sie wollen Eltern dafür gewinnen, mit ihren Kindern an den Vorsorgeuntersuchungen teilzunehmen. Viele Regionen beteiligen sich daher an der landesweiten Aktion der Bundeszentrale für gesundheitliche Ausklärung „Ich geh’ zur U! Und Du?“. Für den Bedarfsfall sollten aber auch Vorgehensweisen implementiert werden, wenn sich Sorgeberechtigte ihrer Fürsorgepflicht entziehen, um die Rechte und den Schutz von Kindern zu stärken. Parallel müssen Kinderärzten die Zeit- und Vergütungsressourcen eingeräumt werden, um ihren Verpflichtungen sensibel und umfassend gerecht $\mathrm{zu}$ werden.

In den Fällen, in denen geschlechtsreife Mädchen mit einer ADHS auffällig werden, sollte umgekehrt von den jeweiligen betreuenden Institutionen verstärkt darauf geachtet werden, sie zu effektiven Verhütungsstrategien zu be- 
wegen. Diese können von der „Babybedenkzeit“ über Partnerberatung bis hin zu möglichst verlässlichen Kontrazeptionen reichen. Wie eingangs erwähnt, sind Interventionen zur genaueren sexuellen Aufklärung hierbei in der Regel ohne Wirkung. Institutionen können dabei Schulen, die Einrichtungen des Jugendamtes und medizinische Behandler sein, aber auch motivierte Eltern und andere signifikante Andere. Gleichzeitig gilt es, den zurzeit herrschenden „Familiendiskurs“ in der deutschen Politik zu nutzen, um die Möglichkeiten der Integration von Familie und Ausbildung/Beruf gerade auch für junge Mütter zu verbessern. Dazu gehört die Ausdehnung der institutionellen Betreuungsmöglichkeiten für Kinder, die direkte Unterstützung der Mutter, aber auch die stärkere Ausrichtung der Psychiatrie auf zu behandelnde Dyaden oder Familien.

Wird ein Kind mit psychiatrischen Auffälligkeiten vorstellig, sollte im Rahmen der klinischen Routine auch an eine ADHS-Symptomatik der Eltern gedacht werden, insbesondere dann, wenn die Mutter zum Zeitpunkt der Geburt ihres ersten Kindes noch Jugendliche war. Gleichzeitige Behandlungen in beiden Generationen, Mutter-Kind-Therapien und Elterntrainings mit und ohne medikamentöse Begleitung sollten dann die psychiatrische Behandlung des Zielkindes ergänzen. In diesen Trainings sollte dann verstärkt auf die besonderen Bedürfnisse jüngerer Mütter und Väter eingegangen werden, eventuelle Nachreifungen bei den Eltern sollten in die Interventionsziele aufgenommen werden.

Auf diese Weise würde nicht nur den Patientinnen und Kindern geholfen, sondern weit reichende volkswirtschaftliche Folgekosten könnten vermieden werden.

\section{Literatur}

Alexander CS, Guyer B. Adolescent pregnancy: occurrence and consequences. Pediatr Ann 1993; 22(2): 85-8.

Ambadekar NN, Khandait DW, Zodpey SP, Kasturwar NB, Vasudeo ND. Teenage pregnancy outcome: a record based study. Indian I Med Sci 1999; 53 (1): 14-7.

Archie CL, Anderson MM, Gruber EL. Positive smoking history as a preliminary screening device for substance use in pregnant adolescents. Pediatr adolesc gynecol 1997; 10(1): 13-7.

Barchmann R (2008). Unveröffentlichtes Manuskript zur Dissertation: Schwangerschaft minderiähriger Mütter - eine Risikoschwangerschaft? Aus dem Projekt Bohne-Suraj S, Reis 0, Gerber B, Häßler F. „Bedingungen und Folgen minderiähriger Mutterschaft" - der Klinik für Psychiatrie, Neurologie, Psychosomatik und Psychotherapie im Kindes- und Jugendalter der Universität Rostock und der Universitätsfrauenklinik am Klinikum Südstadt Rostock.

Barkley RA, Fischer M, Smallish L, Fletcher K. Young adult follow up of hyperactive children: antisocial activities and drug use. I child psychol psychiatry 2004; 45(2): 195-211.

Barnet B, Joffe A, Duggan AK, Wilson MD, Repke JT. Depressive symptoms, stress, and social support in pregnant and postpartum adolescents. Arch Pediatr Adoles Med 1996; 150: 64-69.

Barnet B, Duggan AK, Wilson MD, Joffe A. Association between postpartum substance abuse and depressive symptoms, stress, and social support in adolescent mothers. Pediatrics 1995; 96 (4): 659-66.

Bekker EM, Bocker KBE, van Hunsel F, van den Berg MC, Kenemans JL. Acute effects of nicotine on attention and response inhibition. Pharmacol Biochem Behav 2005; 82(3): 539-548. 
Bertram H. Zur Lage der Kinder in Deutschland: Politik für Kinder als Zukunftsgestaltung. Innocenti Working Paper No. 2006-02. Florence: UNICEF Innocenti Research Centre 2006.

Biederman I, Ball SW, Monuteaux MC, Mick E, Spencer TJ, McCreary M, Cote M, Faraone SV. New Insights Into the Comorbidity Between ADHD and Major Depression in Adolescent and Young Adult Females. I Am Acad Child Adolesc Psychiatry. Feb 2008 [Epub ahead of print].

Billari CF, Dimiter P. Lernen und Familie gründen schließen sich nicht aus. Bildungsbeteiligung und Übergang zur Mutterschaft in Westeuropa. In: Hoern JM, Vaupel JW (Hrsg.). Demografische Forschung. Aus erster Hand. Max-Planck-Institut für demografische Forschung, Rostock. 2006; 3(1). www.demografie-forschung.de.

Birkeland R, Thompson JK, Phares V. Adolescent motherhood and postpartum depression. I Clin Child Adolesc Psychol 2005; 34 (2): 292-300.

Boardman LA, Allsworth I, Phipps MG, Lapane KL. Risk factors for unintended versus intended rapid repeat pregnancies among adolescents. J Adolesc Health 2006; 39(4): 597.

Briscoe-Smith AM, Hinshaw SP. Linkages between child abuse and attention-deficit/hyperactivity disorder in girls: behavioral and social correlates. Child Abuse Negl. 2006; 30(11): 1239-55.

Bundesagentur für Arbeit. 2004. http://www1.arbeitsamt.de/hst/services/statistik/200312/iiia4/multijz_ heftd.pdf. 21.April 2004.

Bundeszentrale für gesundheitliche Aufklärung (BZgA, Hrsg.) Jugendsexualität. Wiederholungsbefragung von 14-bis 17-Jährigen und ihren Eltern. Ergebnisse der Repräsentativbefragung aus 2001, Köln: BZgA 2001.

Bundeszentrale für gesundheitliche Aufklärung (BZgA, Hrsg.). Projekt „Ich geh’ zur U! Und Du?“ http://www. ich-geh-zur-u.de/ 09.04.2008.

Button TM, Thapar A, Mc GaffinP. Relationship between antisocial behaviour, attention-deficit hyperactivity disorder and maternal prenatal smoking. Br | Psychiatry 2005; 187: 155-60.

Camp BW. Adolescent mothers and their children: changes in maternal characteristics and child developmental and behavioral outcome at school age. J Dev Behav Pediatr 1996; 17(3): 162-9.

Coley RL, Chase-Lansdale PL. Adolescent pregnancy and parenthood. Recent evidence and future directions. Am Psychol 1998; 53 (2): 152-66.

Derks EM, Hudziak II, Boomsma DI. Why more boys than girls with ADHD receive treatment: a study of Dutch twins. Twin Res Hum Genet 2007; 10(5): 765-70.

Deutsche Gesellschaft für Kinder- und Jugendpsychiatrie und Psychotherapie u. a. (Hrsg.): Leitlinien zur Diagnostik und Therapie von psychischen Störungen im Säuglings-, Kindes- und Jugendalter. Regulationsstörungen im Säuglings- und Kleinkindalter (0-3 lahre; F98.2 u. a.). 3. überarbeitete Auflage. Köln: Deutscher Ärzte Verlag 2007 a.

Deutsche Gesellschaft für Kinder- und Jugendpsychiatrie und Psychotherapie u. a. (Hrsg.): Leitlinien zur Diagnostik und Therapie von psychischen Störungen im Säuglings-, Kindes- und Jugendalter. Hyperkinetische Störungen (F90). 3. überarbeitete Auflage. Köln: Deutscher Ärzte Verlag 2007 b.

Ebert D, Krause J, Roth-Sackenheim C und das Expertenkomitee. ADHS im Erwachsenenalter - Leitlinien auf der Basis eines Expertenkonsens mit Unterstützung der DGPPN. Nervenarzt 2003; 10: 939-946.

Elkins II, McGue M, lacono WG. Prospective effects of attention-deficit/hyperactivity disorder, conduct disorder, and sex on adolescent substance use and abuse. Arch Gen Psychiatry 2007; 64(10): 1145-52.

Fergusson DM, Woodward LI. Maternal Age and Psychosocial Outcomes in Early Adulthood. I Child Psychol Psychiat 1999; 43 (3): 479-89.

Figueiredo B, Bifulco A, Pacheco A, Costa R, Magarinho R. Teenage pregnancy, attachment style, and depression: a comparison of teenage and adult pregnant women in a portuguese series. Attach and Hum Dev 2006; 8(2): 123-138.

Figueiredo B, Pacheco A, Magarinho R. Adolescent and adult pregnant women: different risk circumstances? Acta Med Port 2005; 18 (2): 97-105.

Flory K, Molina BS, Pelham WE Ir, Gnagy E, Smith B: Childhood ADHD predicts risky sexual behavior in young adulthood. I Clin Child Adolesc Psychol. 2006 Dec; 35(4): 571-7.

Fracassi S. Unveröffentlichtes Manuskript zur Dissertation: Schwangerschaft minderiähriger Mütter - Ist die Risikobelastung ein Effekt des Alters oder bedingt durch Bildungsstand, Partnerstatus und/oder Tabakkonsum? Eine Matched-Pairs-Studie an Erstgebärenden der Jahrgänge 2000-2004. Aus dem Projekt: Bohne-Suraj S, Reis 0 , Gerber B, Häßler F. „Bedingungen und Folgen minderjähriger Mutterschaft“ der Klinik 
für Psychiatrie, Neurologie, Psychosomatik und Psychotherapie im Kindes- und Jugendalter der Universität Rostock und der Universitätsfrauenklinik am Klinikum Südstadt Rostock.

Friedrich M, Remberg A. Wenn Teenager Eltern werden ... Lebenssituationen jugendlicher Schwangerer und Mütter sowie jugendlicher Paare mit Kind. In: Bundeszentrale für gesundheitliche Aufklärung (Hrsg.). Forschung und Praxis der Sexualaufklärung und Familienplanung. Bd. 25. Köln: 2005.

Fuemmeler BF, Kollins SH, McClernon F). Attention deficit hyperactivity disorder symptoms predict nicotine dependence and progression to regular smoking from adolescence to young adulthood. I Pediatr Psychol 2007; 32(10): 1203-1213.

Gama SG, Szwarcwald CL, Leal Md Mdo C. Pregnancy in adolescence, associated factors, and perinatal results among low-income post-partum women. Cad Saude Publica 2002; 18 (1): 153-61.

Garst A. Diskofieber und Muttersorgen - Wenn 14-Jährige Kinder kriegen. In: Fachtagung zu jugendlichen Elternschaften am 10. Oktober 2002: „Sie ist doch selber noch ein halbes Kind ..." im Auftrag der BZgA, pro familia Schleswig-Holstein und des MJFJF Schleswig-Holstein. 2003.

Gaub M, Carlson CL. Gender differences in ADHD: a meta-analysis and critical review. I Am Acad Child Adolesc Psychiatry 1997; 36(8): 1036-45.

Gillmore MR, Gilchrist L, Lee I, Oxford ML. Women who gave birth as unmarried adolescents: trends in substance use from adolescence to adulthood. J Adolesc Health 2006; 39(2): 237-43.

Gilbert WM, Jandial D, Field NT, Bigelow P, Danielsen B. Birth outcomes in teenage pregnancies. J Matern Fetal Neonatal Med 2004; 16: 265-270.

Gisselmann MD. Education, infant mortality, and low birth weight in Sweden 1973-1990: emergence of the low birth weight paradox. Scandinavian I of Public Health 2005; 33: 65-71.

Goldenberg P, Figueiredo MC, Silva RS. Adolescent prenatal care, perinatal outcome in Monte Carlos. Cad Saude Publica. 2005; 21 (4): 1077-86.

Goonewardene IM, Deeyagaha W. Adverse Effect on Teenage Pregnancy. Ceylon Med I 2005; 50(3): 116-20.

Graham P (ed.). Child Psychiatry. A developmental approach. Oxford: Oxford University Press 1991.

Haerty A. Schwangerschaft bei Jugendlichen: Erfahrungen aus Großhadern - Internationaler Vergleich. Münchener Symposium für Kinder- und Jugendgynäkologie. 23.-25.10.2003. http://www.kindergynaekologie. de/html/symp2003_6.html. (05.04.2008).

Häußler-Sczepan M, Wienholz S, Michel M. Teenager-Schwangerschaften in Sachsen. Angebote und Hilfebedarf aus professioneller Sicht. In: Bundeszentrale für gesundheitliche Aufklärung (Hrsg.). Forschung und Praxis der Sexualaufklärung und Familienplanung. Bd. 26. Köln: 2005.

Hamada H, Zaki A, Nejjar H, Filali A, Chraibi C, Bezad R, Alaoui MT. Pregnancy and delivery in adolescents: characteristics and profile of 311 cases. I Gynecol Obstet Biol Reprod 2004; 33 (7): 607-14.

Harner HM. Childhood sex abuse, teenage pregnancy, and partnering with adult men: Exploring the relationship. J of Psychosocial Nursing 2005; 43 (8): 20-8.

Hediger ML, Scholl TO, Schall II, Krueger PM. Young maternal age and preterm labor. Ann Epidemiol 1997; 7 (6): 400-6.

Hillis SD, Anda RF, Dube SR, Felitti V], Marchbanks PA, Marks JS. The association between adverse childhood experiences and adolescent pregnancy, long-term psychosocial consequences, and fetal death. Pediatrics. 2004; $113(2): 320-7$.

Hinshaw SP. Preadolescent girls with attention-deficit/hyperactivity disorder: I. Background characteristics, comorbidity, cognitive and social functioning, and parenting practices. I Consult Clin Psychol 2002; 70(5): 1086-98.

Hinshaw SP, Owens EB, Sami N, Fargeon S. Prospective follow-up of girls with attention-deficit/hyperactivity disorder into adolescence: Evidence for continuing cross-domain impairment. I Consult Clin Psychol 2006; 74(3): 489-99.

Hofferth SL, Reid L. Early childbearing and children's achievement and behaviour over time. Perspect Sex Reprod Health 2002; 34 (1): 41-9.

Holub CK, Kershaw TS, Ethier KA, Lewis JB, Milan S, Ickovics IR. Prenatal and parenting stress on adolescent maternal adjustment: identifying a high-risk subgroup. Matern Child Health I 2007; 11(2): 153-9.

Humphreys C, Garcia-Bournissen F, Ito S, Koren G. Exposure to attention deficit hyper-activity disorder medications during pregnancy. Can Fam Physician 2007; 53(7): 1153-5.

Hurrelmann K, Albert M in Arbeitsgemeinschaft mit Infratest Sozialforschung. 14. Shell Jugendstudie. Ju- 
gend 2002 - Zwischen pragmatischem Idealismus und robustem Materialismus. Frankfurt a. M.: Fischer Taschenbuch 2002.

Jaffee S, Caspi A, Moffitt TE, Belsky J, and Silva P. Why are children born to teen mothers at risk for adverse outcomes in young adulthood? Results from a 20-year longitudinal study. Dev. Psychopathol. 2001; 13 (2): 377-97.

Kaeppler C. Familienbeziehungen bei hyperaktiven Kindern im Behandlungsverlauf. Kindheit und Entwicklung 2005; 14(1): 21-29.

Kaiser MM, Hays B]. Health-risk behaviours in a sample of first time pregnant adolescents. Public Health Nurs 2005; 22(6): 483-493.

Keskinoglu P, Bilgic N, Picakciefe M, Giray H, Karakus N, Gunay T. Perinatal outcomes and risk factors of Turkish adolescent mothers. I Pediatr and Adolesc Gynecol 2007; 20(1): 19-24.

Kirchengast $S$. Wenn Mädchen Mütter werden. Teenagerschwangerschaften - eine biologisches oder soziales Problem? In: Alt KW \& Kemkes-Grottenthaler A (Hg.). Kinderwelten. Köln, Weimar, Wien: Böhlau Verlag 2002.

Kokotailo PK, Langhough RE, Cox NS, Davidson SR, Fleming MF: Cigarette, alcohol and other drug use among small city pregnant adolescents. Adolesc Haelth. 1994,15 (5): 366-73.

Kuchenbecker C. Schwangerschaft und Geburt bei 13- bis 16-jährigen Teenagern. Münchener Symposium für Kinder- und Jugendgynäkologie. 23.-25.10.2003. http://www.kindergynaekologie.de/html/symp2003_7. html. (05.04.2008).

Laucht M, Esser G, Schmidt M. Psychisch auffällige Eltern - Risiken für die kindliche Entwicklung im Säuglingsund Kleinkindalter? ZfF 1992 a; 4: 22-48.

Laucht M, Esser G, Schmidt MH, Ihle W, Löffler W, Stöhr RM, Weindrich D und Weinel H: „Risikokinder“: Zur Bedeutung biologischer und psychosozialer Risiken für die kindliche Entwicklung in den ersten beiden Lebensjahren. Prax Kinderpsychol Kinderpsychiat 1992 b; 41: 274-85.

Laucht M, Schmidt MH. Maternal smoking during pregnancy: risk factor for ADHD in the offspring? Z Kinder Jugendpsychiatr Psychother 2004; 32(3): 177-85.

Laue E. Schwangerschaftsabbrüche und Geburten minderjähriger Schwangerer - die amtliche Statistik. BZgA Forum Sexualaufklärung und Familienplanung 2004; 4: 3-9.

Lee C, Gramotnev H. Predictors and outcomes of early motherhood in the Australian Longitudinal Study on Women's Health. Psychol Health Med. 2006; 11(1): 29-47.

Lee SS, Hinshaw SP. Predictors of adolescent functioning in girls with attention deficit hyperactivity disorder (ADHD): the role of childhood ADHD, conduct problems, and peer status. I Clin Child Adolesc Psychol. 2006;35(3): 356-68.

Malamitsi-Puchner A, Boutsikou T. Adolescent pregnancy and perinatal outcome. Pediatr Endocrinol Rev 2006; $3(1): 170-171$.

Markovitz BP, Cook R, Flick LH, Leet TH. Socioeconomic factors and adolescent pregnancy outcomes: distinctions between neonatal and post neonatal deaths? BMC Public Health 2005; 5(79): 1-7.

Martin A, Ruchkin V, Caminis A, Vermeiren R, Henrich CC, Schwab-Stone M. Early to bed: a study of adaptation among sexually active urban adolescent girls younger than age sixteen. I Am Acad Child Adolesc Psychiatry 2005; 44 (4): 358-67.

Mathews T), Menacker F, MacDorman MF. Infant mortality statistics from the 2001 period linked birth/infant death data set. Natl Vital Stat Rep 2003; 52(2): 1-27.

Maughan B, Lindelow M: Secular change in psychosocial risks: the case of teenage motherhood. Psychol Med. 1997; 27 (5): 1129-44.

Maynard RA. The study, the context, and the findings in brief. In: Maynard RA (ed.). Kids having Kids. Economic costs and social consequences of teen pregnancy. Washington DC: The Urban Institute Press 1996.

McCue Horwitz S, Klerman LV, Sung Kuo H, Jekel F). Intergenerational transmission of school-age parenthood. Fam Plann Perspect 1991; 23: 168-177.

Menacker F, Martin JA, MacDorman MF, Ventura SJ. Births to 10-14 year-old mothers, 1990-2002: trends and health outcomes. Natl Vital Stat Rep 2004; 53 (7): 1-18.

Meyer-Probst B, Teichmann H (Hrsg.). Risiken für die Persönlichkeitsentwicklung im frühen Kindesalter. Leipzig: Thieme 1984 . 
Moessle T, Pfeiffer C, Kleimann M. Mediennutzung von Kindern und Jugendlichen: Stellenwert für Schule, Familie und Freizeit. Zeitschrift für Literaturwissenschaft und Linguistik 2007; 146: 47-66.

Moffit TE and the E-Risk Study team. Teen-aged mothers in contemporary Britain. I of Child Psychol and Psychiatry 2002; 43 (6): 727-42.

Monuteaux MC, Faraone SV, Michelle Gross L, Biederman J. Predictors, clinical characteristics, and outcome of conduct disorder in girls with attention-deficit/hyperactivity disorder: a longitudinal study. Psychol Med 2007; 37(12): 1731-41.

Morash M, Rucker L. An exploratory study of the connection of mother's age at childbearing to her children's delinquency in four data sets. Crime and delinquency 1989; 35 (1): 45-93.

Murray C, Johnston C. Parenting in mothers with and without attentiondeficit/hyperactivity disorder. J Abnorm Psychol 2006; 115(1): 52-61.

Nagin DS, Tremblay RE. Parental and early childhood predictor of persistent physical aggression in boys from kindergarten to high school. Arch Gen Psychiatry 2001; 58: 389-94.

Nagin DS, Pogarsky G, Farrington DP. Adolescent mothers and the criminal behaviour of their children. Law and Society Review 1997; 31(1): 137-162.

Neuhaus C. www.ads-adhsfundgrube.de/PDF/hoerdatei/2_4_d_Frau-Neuhaus-Teil 8 „Mütter mit und ohne ADHS“. Joseph Kennedy Kreatives Lernen 2006. 08.04.2008.

Osthoff R. Schwanger werd' ich nicht alleine ... Ursachen und Folgen ungeplanter Teenager-Schwangerschaften., Landau: Knecht 1999.

Osthoff R. Wenn Mädchen Mütter werden - Probleme und Bewältigungsversuche betroffener Teeanger. In: Deutsche Gesellschaft für Geschlechtserziehung e.V. (Hrsg.). Informationen zur Sexualpädagogik und Sexualerziehung. Heft 3/4 23. Jahrgang, Bonn 2000.

Perry RL, Mannino B, Hediger ML, Scholl TO. Pregnancy in early adolescence: Are there obstetric risks? ) Matern Fetal Med 1996; 5 (6): 333-9.

Plöckinger B, Ulm MR, Chalubinski K, Schaller A. Wenn Kinder "Kinder kriegen“- Reproduktionsbiologische Probleme bei Mädchen zwischen 11 und 15 lahren. Geb Fra 1996; 56: 248-51.

Potter AS, Newhouse P. Effects of acute nicotine administration on behavioral inhibition in adolescents with attention-deficit/hyperactivity disorder. Psychopharmacol 2004; 176(2): 182-194.

Reichel CM, Linkugel JD, Bevins R. Nicotine as a conditioned stimulus: Impact of attention-deficit/hyperactivity disorder medications. Exp Clin Psychopharmacol 2007; 15(5): 501-509.

Remberg A, Weiser S. Wie konnte das passieren? Schwangerschaft im Jugendalter. In: pro familia Magazin 03/2003. Jugend und Sexualität.

Rucklidge IJ, Tannock R. Psychiatric, psychosocial, and cognitive functioning of female adolescents with ADHD. I Am Acad Child Adolesc Psychiatry 2001; 40(5): 530-40.

Saewyc EM, Magee LL, Pettingell SE. Teeanage pregnancy and associated risk behaviors among sexually abused adolescents. Perspect Sex Reprod Health 2004; 36 (3): 98-105.

Sameroff A. Identifying Risk and Protective Factors for Healthy Child Development. In: Dunn I \& Clarke-Stewart A (eds.). Families count: Effects on child and adolescent development. New York: Cambridge University Press 2006.

Seamark Cl, Gray D|. Teenagers and risk taking, pregnancy and smoking. Br I Gen Pract 1998; 48 (427): 985-6.

Schöning I. „Babys sind nicht immer so süß wie sie aussehen.“ Das Projekt „Babybedenkzeit“. In: BZgA (Hrsg.). Forum Sexualaufklärung und Familienplanung 2004; 4: 32-35.

Scholl TO, Hediger ML, Belsky DH: Prenatal care and maternal health during adolescent pregnancy: a review and metaanalysis. I Adolesc Health 1994; 15 (6): 444-56.

Shaw M, Lawlor DA, Najman IM. Teenage children of teenage mothers: psychological, behavioural and health outcomes from an australian prospective longitudinal study. Soc Sci Med. 2006; 62 (10): 2526-39.

Statistisches Bundesamt 2005. www.destatis.de.

Taylor DI, Chavez GF, Adams El, Chabra A, Shah RS. Demographic characteristics in adult paternity for first births to adolescents under 15 years of age. I of Adolescent Health 1999; 24: 251-58.

Trautmann-Villalba P, Gerhold M, Laucht M, Schmidt MH. Early motherhood and disruptive behaviour in the school-age child. Acta Paediatr 2004; 93(1):120-5. 


\section{ADHS und Teenager-Mutterschaft}

Viertler A (2008). Manuskript zur Dissertation: Medizinische und psychosoziale Risiken bei Schwangerschaften im minderjährigen Alter. Aus dem Projekt: Bohne-Suraj S, Reis 0, Gerber B, Häßler F. „Bedingungen und Folgen minderjähriger Mutterschaft" der Klinik für Psychiatrie, Neurologie, Psychosomatik und Psychotherapie im Kindes- und Jugendalter der Universität Rostock und der Universitätsfrauenklinik am Klinikum Südstadt Rostock.

Wakschlag LS, Pickett KE, Cook E |r, Benowitz NL, Leventhal BL. Maternal Smoking during pregnancy and severe antisocial behaviour in offspring: A review. Am | Public Health 2002; 92: 966-974.

Walcher W, Petru E, Tscherne G. Change in obstetrical risk in adolescent primiparous patients - a comparative study. Gynäkol Rundsch 1989; 29 (2): 321-23.

Wanzeck-Sielert C. Sexualpädagogische Hypothesen im Kontext von Jugendkultur - und Sexualforschung. In: BZgA (Hrsg.): Forum Sexualaufklärung und Familienpanung. Jugendkulturen. Köln: 2002.

Whitman TL, Borkowski JG, Keogh DA, Weed K. Intervowen lives. Adolescent mothers and their children, Mahwah, N. J.: Erlbaum 2001.

Wienholz S. Sexualität und Teenager-Schwangerschaften - Ausgewählte Ergebnisse der Vorsorgesituation von Teenagermüttern. In: Gesundheit Berlin (Hrsg.). Dokumentation 12. Bundesweiter Kongress Armut und Gesundheit vom 1./2. Dezember 2006. Berlin: 2007. http://www.gesundheitberlin.de/download/Wienholz.pdf. 08.04.2008.

Yilidrim Y, Inal MM, Tinar S. Reproductive and obstetric characteristic of adolescent pregnancies in turkish women. Pediatr Gynecol 2005; 18(4): 249-53.

Zeteroglu S, Sahin I, Gol K. Cesaren delivery rates in adolescent pregnancy. Eur I Contracept Reprod Health Care. 2005; 10 (2): 119-22.

Ziegenhain U. Förderung von Beziehungs- und Erziehungskompetenzen bei jugendlichen Müttern. Prax Kinderpsychol Kinderpsychiatr 2007; 56(8): 660-75.

Ziegenhain U, Derksen B, Dreisörner R. Frühe Elternschaft: Jugendliche Mütter und ihre Kinder. Monatsschr Kinderheilkd 2003; 151: 608-12. 